\title{
Efficacy of ultrasound-guided transversus abdominis plane block in breast reconstruction surgery with abdominal myocutaneous flap
}

\author{
Ana Luísa Afonso ${ }^{1 *}$, Luís Pereira ${ }^{2}$, Joana Correia ${ }^{1}$, Mónica Ferreira ${ }^{1}$, Nuno Lareiro ${ }^{3}$, Joana \\ Filipa Paulo ${ }^{1}$, Germano Cardoso ${ }^{4}$, Luís Botelho Antunes ${ }^{5}$, Rui Valente ${ }^{4}$ \\ Resident in Anaesthesiology ${ }^{I}$, Instituto de Oncologia do Porto, Francisco Gentil, EPE, Porto \\ Portugal, Assitant of Anaesthesiology ${ }^{2}$, Centro Hospitalar do S. João, EPE, Porto Portugal, \\ Resident in Anaesthesiology ${ }^{3}$, Centro Hospitalar Baixo-Vouga, EPE, Aveiro, Portugal, \\ Graduate Assitant in Anaesthesiology ${ }^{4}$, Instituto de Oncologia do Porto, Francisco Gentil, EPE, \\ Porto Portugal, Epidemiologist ${ }^{5}$, Department of Clinical Epidemiology, Instituto de Oncologia \\ do Porto, Francisco Gentil, EPE, Porto Portugal.
}

Background: Ultrasound (US)-guided transversus abdominis plane block (TAPB) provides postoperative analgesic efficacy in abdominal surgery, however evidence of its efficacy in reconstructive breast surgery with transversus rectus abdominis myocutaneous (TRAM) flap is still limited. Our main objective was to assess effectiveness of TAPB in this surgery.

Methods: Observational retrospective study conducted in patients undergoing unilateral breast reconstruction with TRAM (surgery 1) and mastectomy (partial or total) followed by TRAM reconstruction (surgery 2) from April 2015 to June 2017. Two anaesthetic plans were analysed: general balanced anaesthesia (GBA) and combined GBA with bilateral TAPB (GBA+TAPB).

Primary outcomes were total intraoperative administered fentanyl, total consumed morphine in postoperative anaesthesia care unit (PACU) and time between conclusion of surgery and first requested analgesic (analgesia time).

Results: 107 patients were included: 80 submitted to surgery 1 and 27 submitted to surgery 2 . In surgery 1 , intraoperative fentanyl consumption and administered morphine, during the patients' stay at PACU, were lower in GBA+TAPB than in GBA group $(p<0.001)$. A longer analgesia time was identified in GBA+TAPB group $(p=0.026)$.

Considering surgery 1 , at admission at PACU, GBA+TAPB referred lower pain intensity at rest $(\mathrm{p}=0.049)$ and on movement $(\mathrm{p}=0.001)$, as well as at the $4^{\text {th }}$ postoperative hour, at rest $(p=0.005)$ and on movement $(p=0.011)$. Modified Aldrete scores were higher in GBA+TAPB group $(\mathrm{p}=0.009)$.

Regarding surgery 2, globally no significant differences were identified between both groups.

No complications associated to local anaesthetics or TAPB were registered.

Conclusion: US-guided bilateral TAPB resulted in lower opioid consumption and better pain control in unilateral breast reconstruction surgery with TRAM.

Keywords: Transversus abdominis plane; transversus abdominis plane block; ultrasound; breast reconstruction surgery with abdominal flap

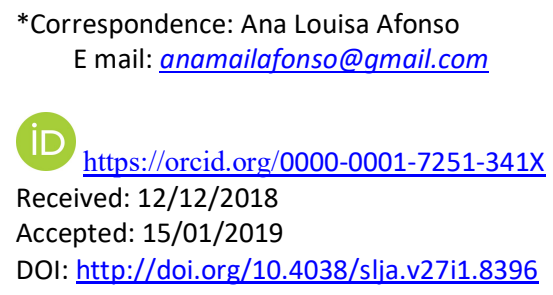

*Correspondence: Ana Louisa Afonso E mail: anamailafonso@gmail.com$$
\text { iD }
$$
https://orcid.org/0000-0001-7251-341X Received: $12 / 12 / 2018$ Accepted: 15/01/2019 DOI: http://doi.org/10.4038/slja.v27i1.8396

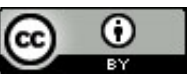

\section{Introduction}

TAPB was first introduced as a landmarkguided technique. ${ }^{1,2}$ This approach was associated with low success rates. Nowadays, the gold standard is US-guided approach, with injection of local anaesthetic between transversus abdominis and internal oblique muscles. ${ }^{3}$ This technique is associated with fewer complications. ${ }^{4,5}$ Various descriptions of TAPB techniques have been published. ${ }^{2,6,7}$ 
An important component of the pain experienced by patients after autologous breast reconstruction with TRAM flap is from the abdominal wall incision. Multimodal analgesia scheme including the performance of TAPB could reduce pain, systemic opioid consumption and their adverse effects. Published literature about TAPB efficacy in this type of surgery is limited. ${ }^{8-10}$

Postoperative pain control can be challenging in the oncological patient. In this group of patients, pain is a complex pathologic process. Mediators liberated by cancer cells sensitize nociceptors, altering endogenous anti nociceptive mechanisms and inducing peripheral and central sensitization. ${ }^{11}$

The goal of this study was to evaluate the efficacy of US-guided TAPB as part of a multimodal analgesia protocol for breast reconstructive surgery with TRAM and mastectomy (partial or total) followed by TRAM reconstruction.

\section{Methods}

This was an observational retrospective study conducted from April 2015 to June 2017 after obtaining our Institutional Ethics Committee's approval.

The study was conducted in the Anaesthesiology Department of an Oncological Institute with extensive experience in breast reconstructive surgery. In 2014, a TAPB protocol was approved (described below) and since then, this locoregional technique has been adopted in this type of surgery.

The inclusion criteria were patients older than 18 years old scheduled for unilateral breast reconstruction surgery with TRAM flap (surgery 1) or to mastectomy (partial or total) followed by TRAM reconstruction (surgery 2). Anaesthetic protocol included general balanced anaesthesia (GBA) or general anaesthesia combined with US guided bilateral TAPB (GBA+TAPB). Only the cases in which TAPB was performed according to our anaesthesiology department's protocol and systemic analgesia followed by an institutional protocol for major somatic surgery were studied.
The exclusion criteria included patients suffering from substance abuse (addiction syndrome); body mass index (BMI) equal to or greater than $40 \mathrm{~kg} / \mathrm{m}^{2}$ and patients subjected to other surgeries besides surgery 1 or surgery 2 within the same operative time.

Intraoperative and postoperative electronic records of the included patients were analysed.

In all included cases the monitoring standards of American Society of Anaesthesiologists (ASA) were adopted. Patients were submitted to GBA with oro-tracheal intubation. Intravenous fentanyl was administered at anaesthetic induction $(1-2 \mu \mathrm{g} / \mathrm{kg})$ and during maintenance (bolus of $0.5-1 \mu \mathrm{g} / \mathrm{kg}$ ). Before surgical incision, patients received parecoxib (40 mg) i.v. and 15 minutes before the conclusions of surgery i.v. paracetamol (1000 $\mathrm{mg}$ ) was administered.

In the included cases of the GBA+TAPB group, TAPB was performed by the assigned anaesthetist after anaesthetic induction, before surgical incision. The technique and the administered volume and concentration of local anaesthetic followed the protocol of our anaesthesiology department, as described below:

- Aseptic technique;

- US-guided technique (Toshiba Nemio XG

iStyle Ultrasound Scanner ${ }^{\circledR}$ ), in-plane approach;

Linear $8 \mathrm{MHz}$ frequency probe; $85 \mathrm{~mm}, 21 \mathrm{G}$ needle (Vygon, Echoplex ${ }^{\circledR}$ );

- At the midaxillary line, halfway between costal margin and iliac crest, transversus abdominis, internal and external oblique muscles were identified;

- After aspiration, $10 \mathrm{ml}$ of ropivacaine $0.375 \%$ was administered on each side of abdominal wall, between transversus abdominis and internal oblique muscles. Ultrasound dispersion of local anaesthetic was observed; - Maximum administered ropivacaine dosage was $3 \mathrm{mg} / \mathrm{kg}$.

Postoperative systemic analgesia protocol included i.v. parecoxib (40 mg) $12 \mathrm{hrly}$, i.v. paracetamol $(1000 \mathrm{mg})$ each 6hrly and supplemental i.v. morphine ( $2 \mathrm{mg}$ boluses until a maximum of $6 \mathrm{mg}$ in 1 hour). 
Total number of bolus $(2-5 \mathrm{mg})$ and total amount of consumed morphine during PACU stay, and total number of administered morphine boluses $(2 \mathrm{mg})$ during the first 4 postoperative hours after discharge from PACU and between 4 and 24 postoperative hours were registered.

Records of pain assessment at rest and on movement were checked at admission and at discharge from PACU and at the $4^{\text {th }}$ and $24^{\text {th }}$ postoperative hours. The following 5-point verbal rating scale was used: "no pain", "slight pain", "moderate pain", "severe pain", "excruciating pain".

Analgesia time, defined as the time, in minutes, since the conclusion of surgery until the moment of the first analgesic request, was registered as well as the worst pain assessed at this point. "No pain" classification was attributed to cases that no rescue analgesia was administered during the study's duration.

Primary endpoints were opioid consumption during intraoperative period and in PACU stay, and analgesia time. Secondary outcomes included opioid consumption after discharge from PACU, pain assessment, total modified Aldrete score and incidence of nausea and vomiting.

\section{Statistical analysis}

Continuous variables were assessed for normality using Shapiro-Wilk test. Summary measures were used to describe continuous and categorical variables. All conducted analyses were stratified by type of surgery (surgery 1 vs surgery 2). P values of $<0.05$ were regarded as significant.

Distribution of continuous variables were compared between both groups using nonparametric test Mann-Whitney U. Chi Square or Fisher tests were used when appropriate to verify associations between categorical variables.

The "analgesia time" variable was analysed using Kaplan-Meier curves and the differences between both groups were verified using logrank test.

Software SPSS $®$ (Version 24.0, Chicago, IL) was used.

\section{Results}

One hundred and seven patients were included: 80 submitted to surgery 1 and 27 submitted to surgery 2. No significant differences were found between GBA and GBA+TAPB groups in age, BMI, ASA classification and anaesthesia duration for both surgeries (Table 1). All studied patients were classified as ASA physical status II. According to intraoperative records, propofol was the intravenous anaesthetic used in all included cases and sevoflurane or desflurane were used as volatile maintenance agents. Antiemetic prophylaxis with i.v. ondansetron (4 mg) or droperidol $(1.25 \mathrm{mg})$ or both was administered 15-30mins before conclusion of surgery.

Table 1: Descriptive analysis

\begin{tabular}{|c|c|c|c|c|c|c|c|}
\hline \multirow{2}{*}{\multicolumn{2}{|c|}{ Variables }} & \multicolumn{2}{|c|}{ Surgery 1} & \multirow{2}{*}{$\begin{array}{l}\mathrm{p} \\
\mathrm{va} \\
\text { lu } \\
\mathrm{e}\end{array}$} & \multicolumn{2}{|c|}{ Surgery 2} & \multirow{2}{*}{$\begin{array}{l}\text { p } \\
\text { va } \\
\text { lu } \\
\text { e }\end{array}$} \\
\hline & & GBA & $\begin{array}{l}\text { GBA } \\
+ \\
\text { TAP }\end{array}$ & & GBA & $\begin{array}{l}\text { GBA } \\
+ \\
\text { TAP }\end{array}$ & \\
\hline \multicolumn{2}{|c|}{ Age (in years) } & 50.0 & 52.0 & \multirow{2}{*}{$\begin{array}{l}0 . \\
69 \\
0\end{array}$} & & 48.0 & \multirow{2}{*}{$\begin{array}{l}0 . \\
98 \\
0\end{array}$} \\
\hline Median | & 25-P75 & $\begin{array}{l}\mid 42- \\
56.5\end{array}$ & $\begin{array}{l}\mid 42.5 \\
-55 \mid\end{array}$ & & $\begin{array}{l}\mid 44.5- \\
55 \mid\end{array}$ & $\begin{array}{l}\mid 45- \\
57.5 \mid\end{array}$ & \\
\hline \multirow{2}{*}{\multicolumn{2}{|c|}{$\begin{array}{l}\text { BMI (in kg/m²) } \\
\text { Median |P25-P75| }\end{array}$}} & 26.2 & & \multirow{2}{*}{$\begin{array}{l}0 . \\
54 \\
5\end{array}$} & 25.8 & & \multirow{2}{*}{$\begin{array}{l}0 . \\
98 \\
0\end{array}$} \\
\hline & & $\begin{array}{l}\mid 23.8- \\
27.3 \mid\end{array}$ & $\begin{array}{l}\mid 24.6 \\
- \\
27.7 \mid\end{array}$ & & $\begin{array}{l}\mid 22.3- \\
28.1 \mid\end{array}$ & $\begin{array}{l}\mid 25.6 \\
- \\
28.3 \mid\end{array}$ & \\
\hline $\begin{array}{l}\text { ASA } \\
\text { Physic } \\
\text { al } \\
\text { Status }\end{array}$ & $\|$ & 48 & 32 & - & 18 & 9 & - \\
\hline \multirow{2}{*}{$\begin{array}{l}\text { Volatile } \\
\text { agent }\end{array}$} & $\begin{array}{l}\text { Sevofl } \\
\text { urane }\end{array}$ & 19 & 6 & \multirow{2}{*}{$\begin{array}{l}0 . \\
05 \\
4\end{array}$} & 4 & 1 & \multirow{2}{*}{$\begin{array}{l}0 . \\
63 \\
6\end{array}$} \\
\hline & $\begin{array}{l}\text { Desflur } \\
\text { ane }\end{array}$ & 29 & 26 & & 14 & 8 & \\
\hline \multirow{2}{*}{$\begin{array}{l}\mathrm{AE} \\
\text { Prophy } \\
\text { laxis }\end{array}$} & 1 & 1 & 1 & \multirow{2}{*}{$\begin{array}{l}1 . \\
00 \\
0\end{array}$} & 1 & 1 & \multirow{2}{*}{$\begin{array}{l}1 . \\
00 \\
0\end{array}$} \\
\hline & 2 & 47 & 31 & & 17 & 8 & \\
\hline \multicolumn{2}{|c|}{$\begin{array}{l}\text { Anaesthesia } \\
\text { duration } \\
\text { (in minutes) } \\
\text { Median |P25-P75| }\end{array}$} & $\begin{array}{l}178 \\
\mid 165- \\
200 \mid\end{array}$ & $\begin{array}{l}190 \\
\mid 175- \\
210 \mid\end{array}$ & $\begin{array}{l}0 . \\
07 \\
2\end{array}$ & $\begin{array}{l}210 \\
\mid 198.8 \\
242.5 \mid\end{array}$ & $\begin{array}{l}195 \\
\mid 170- \\
242 . \\
5 \mid\end{array}$ & $\begin{array}{l}0 . \\
50 \\
3\end{array}$ \\
\hline \multicolumn{2}{|c|}{ Number (n, \%) } & $\begin{array}{l}48 \\
44.9 \%\end{array}$ & $\begin{array}{l}32, \\
29.9 \\
\%\end{array}$ & - & $\begin{array}{l}18, \\
16.8 \%\end{array}$ & $\begin{array}{l}9, \\
8.4 \%\end{array}$ & - \\
\hline
\end{tabular}

Legend: ASA - American Society of Anaesthesiology, AE - Antiemetic. 1 = IV ondansetron (4 mg) or droperidol (1.25 mg); 2 $=\mathrm{IV}$ ondansetron $(4 \mathrm{mg})$ and droperidol (1.25 $\mathrm{mg}$ ).

Considering surgery 1, consumed fentanyl was significantly lower in GBA+TAPB group than in GBA group. Total number of boluses 
and total amount of supplemental morphine were significantly lower in GBA+TAP group.

No statistically significant differences were found in opioid consumption after discharge from PACU. We found that the number of administered boluses of supplemental morphine was below 2 in most patients in both surgeries (Table 2).

Regarding the opioid consumption, no significant differences were detected in surgery 2 .

Table 2: Opioid consumption during intraoperative period, in PACU stay, since discharge from PACU until 4 postoperative hours and between 4 and 24 postoperative hours.

\begin{tabular}{|c|c|c|c|c|c|c|c|c|}
\hline \multirow{2}{*}{\multicolumn{3}{|c|}{ Variables }} & \multicolumn{2}{|c|}{ Surgery 1} & \multirow{2}{*}{$\begin{array}{l}P \\
\text { va } \\
\text { lu } \\
\text { e } \\
\end{array}$} & \multicolumn{2}{|c|}{$\begin{array}{l}\text { Surgery } 2 \\
\end{array}$} & \multirow{2}{*}{$\begin{array}{l}\mathrm{P} \\
\text { val } \\
\text { ue }\end{array}$} \\
\hline & & & $\underline{\text { GBA }}$ & $\begin{array}{l}\text { GBA } \\
\stackrel{ \pm}{ \pm A P} \\
\underline{\text { APP }}\end{array}$ & & $\underline{\text { GBA }}$ & $\begin{array}{l}\text { GBA } \\
\pm \\
\underline{T} \\
\end{array}$ & \\
\hline \multicolumn{3}{|c|}{$\begin{array}{l}\text { Fentanyl consumption* } \\
\text { (in mg/kg) } \\
\text { Median |P25-P75| }\end{array}$} & $\begin{array}{c}0.00 \\
5 \\
\mid 0.00 \\
39- \\
0.00 \\
63 \mid\end{array}$ & $\begin{array}{r}0.0035 \\
\mid 0.0029- \\
0.0044 \mid\end{array}$ & $\begin{array}{l}<0 \\
.0 \\
01\end{array}$ & $\begin{array}{l}0.006 \\
3 \\
\mid 0.004 \\
6- \\
0.007 \\
7 \mid\end{array}$ & $\begin{array}{l}0.004 \\
8 \\
\mid 0.003 \\
5- \\
0.006 \\
6 \mid\end{array}$ & 0.08 \\
\hline \multicolumn{3}{|c|}{$\begin{array}{l}\text { Supplemental morphine } \\
\text { (number of bolus) } \\
\text { Median |P25-P75| }\end{array}$} & $\begin{array}{c}1,0 \\
|0-2|\end{array}$ & $0,0|0-2|$ & $\begin{array}{l}0 . \\
02 \\
1\end{array}$ & $\begin{array}{l}0,0 \mid 0- \\
2 \mid\end{array}$ & $\begin{array}{l}1,0 \mid 1- \\
2 \mid\end{array}$ & $\begin{array}{l}0.14 \\
6\end{array}$ \\
\hline \multicolumn{3}{|c|}{$\begin{array}{c}\begin{array}{c}\text { Total amount of morphine* } \\
\text { (in mg) }\end{array} \\
\text { Median |P25-P75| } \\
\end{array}$} & $\begin{array}{c}2,5 \\
|0-6|\end{array}$ & $0,0|0-4|$ & $\begin{array}{l}0 . \\
03 \\
6\end{array}$ & $\begin{array}{l}0,0 \mid 0- \\
4 \mid\end{array}$ & $\begin{array}{l}4,0 \mid 2- \\
4 \mid\end{array}$ & $\begin{array}{l}0.10 \\
1\end{array}$ \\
\hline \multirow{3}{*}{$\begin{array}{l}\text { Number of } \\
\text { morphine } \\
\text { boluses } \\
\text { (since } \\
\text { discharge } \\
\text { from PACU } \\
\text { until } 4 \\
\text { postoperati } \\
\text { ve hours) } \\
\star \star \star\end{array}$} & \multicolumn{2}{|c|}{$0 \mathrm{n}(\%)$} & $\begin{array}{c}37 \\
(77.1\end{array}$ & $\begin{array}{c}27 \\
(84.4)\end{array}$ & \multirow{3}{*}{$\begin{array}{l}0 . \\
57 \\
1\end{array}$} & $\begin{array}{l}15 \\
(83.3)\end{array}$ & $\begin{array}{l}5 \\
(55.6)\end{array}$ & \multirow{3}{*}{$\begin{array}{l}0.17 \\
5\end{array}$} \\
\hline & $\geq$ & $\begin{array}{c}1 \\
n \\
(\%) \\
\end{array}$ & $\begin{array}{c}10 \\
(20.8 \\
)\end{array}$ & $\begin{array}{c}5 \\
(15.6)\end{array}$ & & $\begin{array}{l}3 \\
(16.7)\end{array}$ & $\begin{array}{l}4 \\
(44.4)\end{array}$ & \\
\hline & 1 & $\begin{array}{c}2 \\
n \\
(\%)\end{array}$ & $\begin{array}{c}1 \\
(2.1)\end{array}$ & 0 & & 0 & 0 & \\
\hline \multirow{3}{*}{$\begin{array}{c}\text { Number of } \\
\text { morphine } \\
\text { boluses } \\
(4-24 \\
\text { postoperati } \\
\text { ve hours) } \\
\star * \star *\end{array}$} & \multicolumn{2}{|c|}{$\begin{array}{c}0 \\
n(\%)\end{array}$} & $\begin{array}{c}37 \\
(77.1 \\
)\end{array}$ & $\begin{array}{c}26 \\
(81.2)\end{array}$ & \multirow{3}{*}{$\begin{array}{l}0 . \\
78 \\
3\end{array}$} & $\begin{array}{l}14 \\
(77.8)\end{array}$ & $\begin{array}{l}6 \\
(66.7)\end{array}$ & \multirow{3}{*}{$\begin{array}{l}0.65 \\
3\end{array}$} \\
\hline & \multirow{2}{*}{$\begin{array}{l}\geq \\
1\end{array}$} & $\begin{array}{c}1 \\
n \\
(\%) \\
\end{array}$ & $\begin{array}{c}9 \\
(18.8 \\
) \\
\end{array}$ & $\begin{array}{c}3 \\
(9.4)\end{array}$ & & $\begin{array}{l}4 \\
(22.2)\end{array}$ & $\begin{array}{l}3 \\
(33.3)\end{array}$ & \\
\hline & & $\begin{array}{c} \\
n \\
(\%)\end{array}$ & $\begin{array}{c}2 \\
(4.1)\end{array}$ & $\begin{array}{c}3 \\
(9.4)\end{array}$ & & 0 & 0 & \\
\hline
\end{tabular}

*intraoperative; ** PACU; *** after discharge from PACU

In surgery 1, longer analgesia time was identified in GBA+TAPB group ( $\mathrm{p}=0.026)$. Considering surgery 2, there wasn't a significant difference between both groups (Figure 1). Upon the first rescue analgesic administration, GBA group referred higher pain intensity with $56.3 \%$ of patients referring "moderate pain" / "intense pain" compared to $25 \%$ in GBA+TAPB group ( $\mathrm{p}=0.006)$.

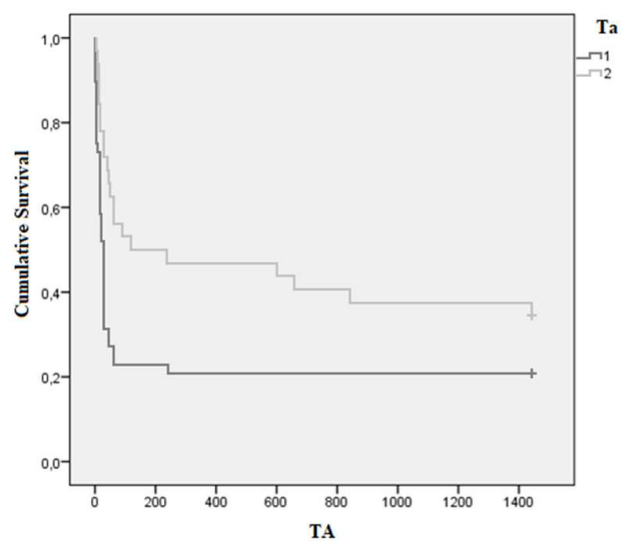

(A)

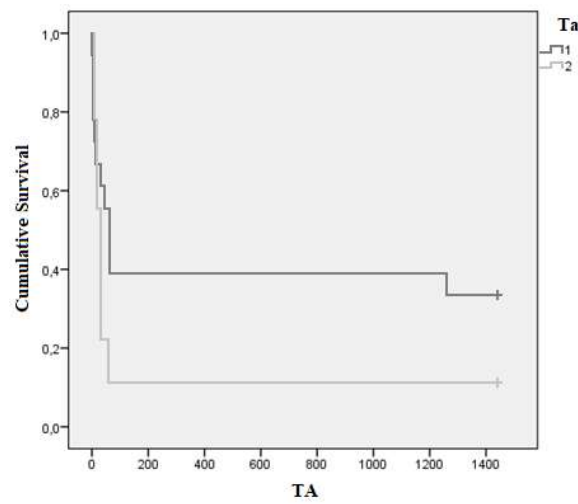

(B)

Figure 1: Survival curve - analgesia time (TA, in minutes). A - surgery 1 ; B- surgery 2. Ta - anaesthetic technique (1 - GBA; 2 $\mathrm{GBA}+\mathrm{TAP})$

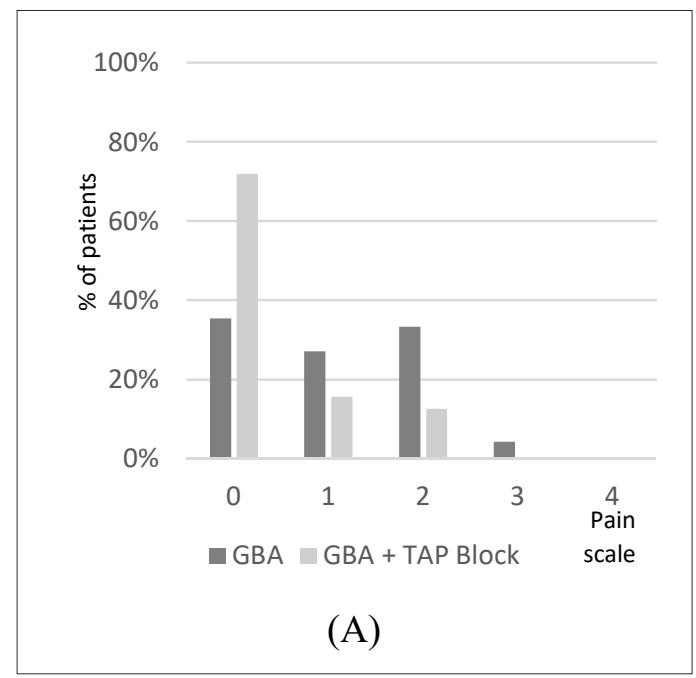


Afonso et al. Sri Lankan Journal of Anaesthesiology: 27(1):59-67(2019)
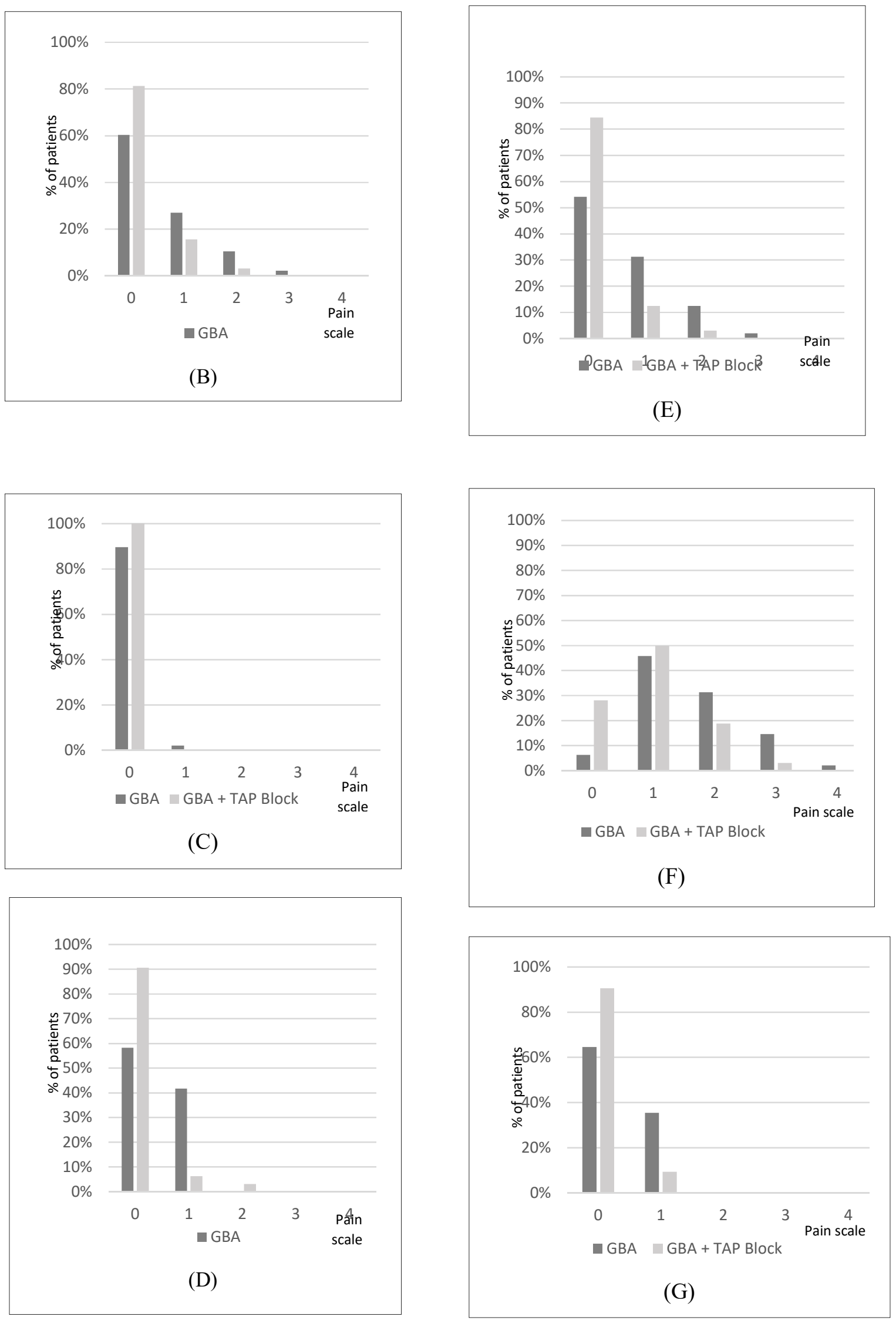


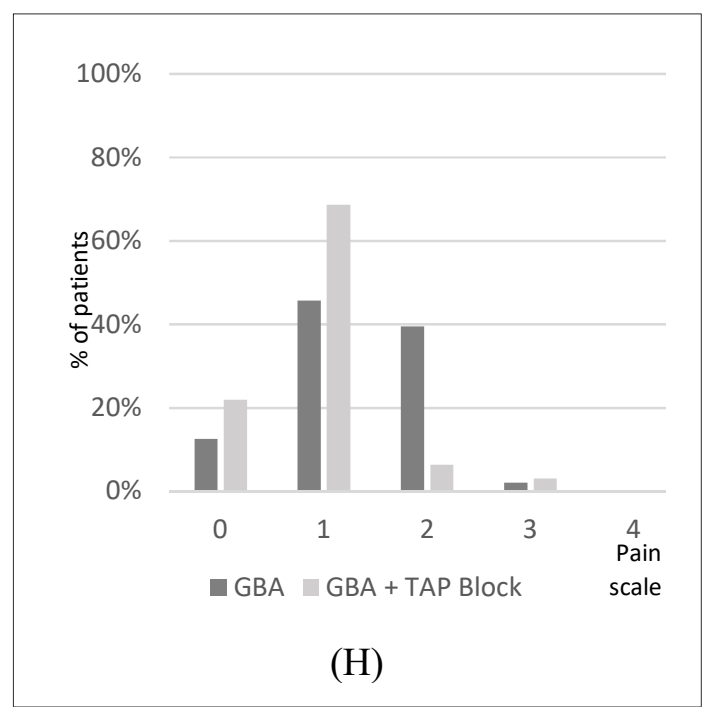

Figure 2 -Distribution of patients according to pain assessment: at admission at PACU, at rest (A) and on movement (B); at discharge from PACU at rest (C) and on movement (D); at $4^{\text {th }}$ postoperative hour at rest $(\mathrm{E})$ and on movement $(\mathrm{F})$ and at $24^{\text {th }}$ postoperative hour at rest $(\mathrm{G})$ and on movement $(\mathrm{H})$. "no pain"- 0 ; "mild pain"1; "moderate pain"- 2; "severe pain" - 3; "excruciating pain" - 4. Statistical analysis executed -0 vs $\geq 1$

Also in surgery 1 , at admission at PACU, $81.3 \%$ in GBA+TAPB group and $60.4 \%$ in GBA group referred "no pain" at rest $(\mathrm{p}=0.049)$. Considering pain on movement, $71.9 \%$ in GBA+TAPB group and $35.4 \%$ in GBA referred "no pain" $(\mathrm{p}=0.001)$. At discharge from PACU, 90.6\% in GBA + TAPB group referred "no pain" on movement compared to $58.3 \%$ in GBA group ( $\mathrm{p}=0.002$ ). No difference was found at rest (Figure 2). No significant differences were detected in surgery 2.

At $4^{\text {th }}$ postoperative hour, in surgery $1,84.4 \%$ in GBA+TAPB group and $54.2 \%$ in GBA group referred "no pain" at rest $(\mathrm{p}=0.005)$. Considering pain on movement, $28.1 \%$ in GBA+TAPB group and $6.3 \%$ in GBA group referred "no pain" $(\mathrm{p}=0.011)$ (Figure 2). Regarding surgery 2, a significant difference was identified only at rest $(\mathrm{p}=0.029)$. In addition, concerning pain on movement,
$93.8 \%$ of patients in GBA group and $71.9 \%$ in GBA+TAPB group referred at least "mild pain" for both surgeries.

At the $24^{\text {th }}$ postoperative hour, in surgery 1 , $90.6 \%$ in GBA+TAPB group and $64.6 \%$ in GBA group referred "no pain" at rest $(\mathrm{p}=0.009)$ (Figure 2).

No significant difference was observed in the recovery time (PACU stay) between groups in both surgeries.

At discharge from PACU, considering surgery 1, GBA+TAPB group presented higher total modified Aldrete score. No significant differences were observed in surgery 2 (Table $3)$.

Table 3: Analysed variables during PACU stay

\begin{tabular}{|c|c|c|c|c|c|c|c|}
\hline \multirow{2}{*}{\multicolumn{2}{|c|}{ Variables }} & \multicolumn{2}{|c|}{ Surgery 1} & \multirow{2}{*}{$\begin{array}{l}P \\
\text { value }\end{array}$} & \multicolumn{2}{|c|}{ Surgery 2} & \multirow{2}{*}{$\begin{array}{l}P \\
\text { value }\end{array}$} \\
\hline & & GBA & $\begin{array}{ll}\text { GBA } \\
\text { IAPB } \\
\end{array}$ & & GBA & $\begin{array}{ll}\text { GBA } & + \\
\text { TAPB } & \end{array}$ & \\
\hline \multicolumn{2}{|c|}{$\begin{array}{l}\text { Length of PACU stay } \\
\text { (in minutes) }\end{array}$} & $45-65$ & $40-68$ & 0.599 & $55-75$ & $50-73$ & 0.499 \\
\hline \multicolumn{2}{|c|}{$\begin{array}{l}\text { Total modified Aldrete } \\
\text { score on admission } \\
\text { Median |P25-P75| }\end{array}$} & $\begin{array}{l}9.0 \\
|8.0-10.0|\end{array}$ & $\begin{array}{l}9.5 \\
|8.0-10.0|\end{array}$ & 0.247 & $\begin{array}{l}9.0 \mid 8.75- \\
10.0 \mid\end{array}$ & $\begin{array}{l}9.0 \\
|8.5-10.0|\end{array}$ & 0.749 \\
\hline \multirow{2}{*}{$\begin{array}{l}\text { Total modified } \\
\text { Aldrete score } \\
\text { at discharge } \\
\text { from PACU }\end{array}$} & $\begin{array}{l}8 / 9 \\
n \\
(\%) \\
\end{array}$ & $\begin{array}{l}21 \\
(43.8 \%) \\
\end{array}$ & $5(15.6 \%)$ & \multirow{2}{*}{0.009} & $\begin{array}{l}7 \\
(38.9 \%) \\
\end{array}$ & $3(33.3 \%)$ & \multirow{2}{*}{1.000} \\
\hline & $\begin{array}{l}10 \\
n \\
(\%) \\
\end{array}$ & $\begin{array}{l}27 \\
(56.2 \%) \\
\end{array}$ & $27(56.2 \%)$ & & $\begin{array}{l}11 \\
(61.1 \%)\end{array}$ & $6(66.7 \%)$ & \\
\hline \multirow{2}{*}{$\begin{array}{l}\text { Consciousness } \\
\text { at admission } \\
\text { (assessed in } \\
\text { Aldrete score) }\end{array}$} & $\begin{array}{l}1 \mathrm{n} \\
(\%)\end{array}$ & $\begin{array}{l}27 \\
(56.3 \%) \\
\end{array}$ & $11(34.4 \%)$ & \multirow{2}{*}{0.055} & $\begin{array}{l}10 \\
(55.6 \%) \\
\end{array}$ & $4(44.4 \%)$ & \multirow{2}{*}{0.695} \\
\hline & $\begin{array}{l}2 \mathrm{n} \\
(\%)\end{array}$ & $\begin{array}{l}21 \\
(43.7 \%) \\
\end{array}$ & $21(65.6 \%)$ & & $\begin{array}{l}8 \\
(44.4 \%) \\
\end{array}$ & $5(55.6 \%)$ & \\
\hline \multirow{2}{*}{$\begin{array}{l}\text { Consciousness } \\
\text { at discharge } \\
\text { (assessed in } \\
\text { Aldrete score) }\end{array}$} & $\begin{array}{l}1 n \\
(\%)\end{array}$ & $\begin{array}{l}5 \\
(10.4 \%)\end{array}$ & $0(0.0 \%)$ & \multirow{2}{*}{0.080} & $\begin{array}{l}2 \\
(11.1 \%)\end{array}$ & $0(0.0 \%)$ & \multirow{2}{*}{0.538} \\
\hline & $\begin{array}{l}2 \mathrm{n} \\
(\%)\end{array}$ & $\begin{array}{l}43 \\
(89.6 \%)\end{array}$ & $\begin{array}{l}32 \\
(100.0 \%)\end{array}$ & & $\begin{array}{l}16 \\
(88.9 \%)\end{array}$ & $\begin{array}{l}9 \\
(100.0 \%)\end{array}$ & \\
\hline
\end{tabular}

No significant differences were found regarding the length of in-hospital stay between both groups.

No differences were identified in regard to nausea or vomiting incidence. In fact, the main registered medical complication in GBA group, considering both surgeries, was constipation. Regarding surgery 1, constipation incidence was $31.3 \%$ in GBA group and $3.1 \%$ in GBA + TAPB group. Considering surgery 2 , constipation incidence was $22.2 \%$ for GBA group and $0 \%$ for GBA + TAPB group.

There were neither reported complications associated with TAPB administration nor clinical cases of local anaesthetic toxicity. 


\section{Discussion}

US-guided TAPB has been showing promising results in abdominal surgery. It has become a well-recognized postoperative analgesia technique. ${ }^{2-5}$

Multimodal analgesia regimens should include techniques that facilitate postoperative pain control, contributing to reduction of incidence of chronic pain. Considering breast reconstructive surgery, a substantial number of patients reported pain-related complaints for longer periods than was expected. ${ }^{12,13}$

Our work is one of the first anaesthesiology studies evaluating bilateral TAPB efficacy in breast reconstructive surgery with TRAM flap. Based on our results and on published studies, an important pain component in this surgery derives from incision in the abdominal donor site, explaining why a peripheral block of abdominal wall, like TAPB, is effective in postoperative pain control. ${ }^{8,9,14}$

In truth, no benefit was detected in patients submitted to mastectomy followed by TRAM reconstruction. In these cases, pain in the mammary area may be higher after mastectomy than after a simple flap inset, justifying the use of a thoracic wall block. ${ }^{9,15,16}$

As verified in other studies, administration of long-acting local anaesthetics that block the sensory nerves to abdominal wall allows a significant reduction of systemic opioid consumption. In previous studies that evaluated TAPB in breast reconstruction with abdominal flap, reduced morphine consumption in the early postoperative period was reported. ${ }^{8,10}$ In our study, reduction of intraoperatively administered fentanyl and immediate postoperative consumption of morphine was identified.

Analgesia time was included in the primary outcomes of our study. It is an important variable to consider when analysing an analgesia scheme's efficacy. ${ }^{5,17}$ At this point, we concluded that TAPB was associated with an increase in time to first analgesia request.

Moreover, results of pain assessment at PACU and at $4^{\text {th }}$ postoperative hour support that the positive effect of bilateral TAPB in TRAM surgery is mainly effective during the first postoperative hours. However, we detected that the incidence of mild, moderate and severe pain was greater on movement, especially in the first four post-operative hours, which is in line with the fact that complete relief of pain on movement is not commonly achieved with local or systemic techniques but through neuraxial techniques. ${ }^{18,19}$ To our knowledge, there aren't any studies comparing epidural with TAPB in TRAM surgery.

Published plastic surgery studies evaluated a continuous TAPB technique and reported good results. ${ }^{8,20}$ A catheter-based technique would allow extended postoperative analgesia decreasing morphine consumption for more than 24 hours.

The reduction in opioid consumption resulted in higher total modified Aldrete score at discharge from PACU. All the patients submitted to TAPB scored 2 in the consciousness component of Aldrete score and most of them held maximum total modified Aldrete scores.

Even though, no significant difference was detected in the incidence of nausea and vomiting during in-hospital stay, we must consider the variability of patients' risk factors and the different number and classes of prophylactically administered antiemetics.

No complications related to TAPB administration were registered. According to literature, we believe that US-guided bilateral TAPB is a safe technique. ${ }^{5}$

The limitations of this study are the reduced number of included patients, the retrospective nature of the study and the fact that this is a relatively novel technique in our daily institutional anaesthetic practice.

\section{Conclusion}

US-guided bilateral TAPB presents positive effects in opioid consumption, analgesia time and pain scores in unilateral breast reconstruction with TRAM but not in mastectomy (partial or total) followed by TRAM reconstruction.

An interesting aspect to study in the future is the correlation between TAPB performance 
and incidence of chronic pain in patients subjected to this surgery.

Conflicts of interest: None.

Financial support: None.

\section{References}

1. Atkinson RS, Rushman GB, Lee JA. A Synopsis of Anaesthesia, 10th ed. Bristol: Wright; 1987.

2. Rafi AN. Abdominal field block: a new approach via the lumbar triangle. Anaesthesia 2001; 56(10): 1024-6. https://doi.org/10.1046/j.13652044.2001.02279-40.x PMid:11576144

3. Hebbard P, Fujiwara Y, Shibata Y, Royse C. Ultrasound-guided transversus abdominis plane (TAPB) block. Anaesth Intensive Care 2007; 35(4): 616-7. PMid: 18020088

4. Ripollés J, Mezquita SM, Abad A, Calvo J. Analgesic efficacy of the ultrasound-guided blockade of the transversus abdominis plane - a systematic review. Braz J of Anesthesiol 2015; 65(4): 255-80. https://doi.org/10.1016/j.bjan.2013.10.014 PMid:26145531

5. Ma N, Duncan JK, Scarfe AJ, Schuhmann S. Clinical safety and effectiveness of transversus abdominis plane (TAPB) block in postoperative analgesia: a systematic review and meta-analysis. J Anesth 2017; 31(3): 432-52.

https://doi.org/10.1007/s00540-017-2323-5 PMid:28271227

6. Hebbard P. Subcostal transversus abdominis plane block under ultrasound guidance. Anesth and Analg 2008; 106(2): 674-75. https://doi.org/10.1213/ane.0b013e318161a $\underline{88 \mathrm{f}}$ PMid: 18227342

7. Faiz SHR, Alebouyeh MR, Derakhshan P, Imani F, Rahimazaden P, Ashtiani MG. Comparison of ultrasound-guided posterior transversus abdominis plane block and lateral transversus abdominis plane block for postoperative pain management in patients undergoing cesarean section: a randomized double-blind clinical trial study. J of Pain Research 2018; 11: 5-9. https://doi.org/10.2147/JPR.S146970 PMid:29296094 PMCid:PMC5741073

8. Zhong $\mathrm{T}$, Ojha M, Bagher S, Butler $\mathrm{K}$, Srinivas C, McCluskey SA et al. Transversus Abdominis Plane Block
Reduces Morphine Consumption in the Early Postoperative Period following Microsurgical Abdominal Tissue Breast Reconstruction: A Double-Blind, PlaceboControlled, Randomized Trial. PlastReconstrSurg Journal 2014; 134(5): 870-8.

https://doi.org/10.1097/PRS.0000000000000613 PMid:25347623

9. Nash H, Khoda B, Heppell S, Turner M. TAPB blocks in breast reconstructions using abdominal wall tissue. Anaesthesia 2011; 66: 750-51 [Correspondence]

https://doi.org/10.1111/j.13652044.2011.06798.x

PMid:21749351

10. Hivelin M, Wyniecki A, Plaud B, Marty J, Lantieri L. Ultrasound-Guided Bilateral Transversus Abdominis Plane Block for Postoperative Analgesia after Breast Reconstruction by DIEP Flap. Plastic and Reconstructive Surgery Journal 2011; 128(1): 44-55. https://doi.org/10.1097/PRS.0b013e3182174090 PMid:21701318

11. Schmidt BL, Hamamoto DT, Simone DA, Wilcox GL. Mechanism of Cancer Pain. MolInterv 2010; 10(3): 164-78. https://doi.org/10.1124/mi.10.3.7 PMid:20539035 PMCid:PMC2895277

12. Kulkarni AR, Pusic AL, Hamill JB, Kim HM, Qi J, Wilkins EG et al. Factors associated with acute postoperative pain following breast reconstruction. JPRAS Open 2017; 11: 1-13. https://doi.org/10.1016/j.jpra.2016.08.005 PMid:28713853 PMCid:PMC5507622

13. Hickey OT, Nugent NF, Burke SM, Hafeez P, Mudrakouski AL, Shorten GD. Persistent pain after mastectomy with reconstruction. J ClinAnesth 2011; 23: 482-88. https://doi.org/10.1016/j.jclinane.2011.01.009 PMid:21911195

14. Albornoz CR, Bach PB, Pusic AL, McCarthy CM, Mehrara BJ, Disa JJ et al. The Influence of Sociodemographic Factors and Hospital Characteristics on the Method of Breast Reconstruction, Including Microsurgery: A U.S. Population-Based Study. PlastReconstrSurg 2012; 129 (5): 1071-79. https://doi.org/10.1097/PRS.0b013e31824a 29c5

PMid:22544091

15. Kamiya $\mathrm{Y}$, Hasegawa $\mathrm{M}$, Yoshida $\mathrm{T}$, Takamatsu M, Koyama Y. Impact of pectoral nerve block on postoperative pain and quality of recovery. Eur J Anaesthesiol 2018; 35(3): 215-23.

PMid:29227351 
Afonso et al. Sri Lankan Journal of Anaesthesiology: 27(1):59-67(2019)

16. Boehmler JH, Venturi ML, Nahabedian MY. Decreased Narcotic Use With an Implantable Local Anaesthetic Catheter After Deep Inferior Epigastric Perforator Flap Breas Reconstruction. An PlastSurg 2009; 62(6): 618-20.

https://doi.org/10.1097/SAP.0b013e31817fe61c PMid:19461271

17. Ersayli DT, Gurbet A, Bekar A, Uckunkaya $\mathrm{N}$, Bilgin $\mathrm{H}$. Effects of Perioperatively Administered Bupivacaine and Bupivacaine-Methylprednisolone on Pain after Lumbar Discectomy. Spine (Phila Pa) 2006; 31(19): 2221-6.

https://doi.org/10.1097/01.brs.0000232801.19965.a0 PMid:16946657

18. Srikandarajah S, Gilron I. Systematic review of movement-evoked pain versus pain at rest in postsurgical clinical trials and meta analyses: A fundamental distinction requiring standardized measurement. Pain 2011; 152(8): 1734 - 9 .

https://doi.org/10.1016/j.pain.2011.02.008 PMid:21402445

19. Iyer SS, Bavishi H, Mohan CV, Kaur N. Comparison of Epidural Analgesia with Transversus Abdominis Plane Analgesia for Postoperative Pain Relief in Patients Undergoing Lower Abdominal Surgery: A Prospective Randomized Study. Anaesth Essays Res 2017; 11(3): 670-5.

https://doi.org/10.4103/0259-1162.206856 PMid:28928569 PMCid:PMC5594788

20. Giordano S, Verajankorva E, Koskivuo I, Suominen E. Effectiveness of local anaesthetic pain catheters for abdominal donor site analgesia in patients undergoing free lower abdominal flap breast reconstruction: A meta-analysis of comparative studies. J PlastSurg Hand Surg 2013; 47(6): 428-33.

https://doi.org/10.3109/2000656X.2013.788508 